Open Peer Review on Qeios

\title{
ORAL HEALTH STATUS IN A COHORT OF 114 CANCER PATIENTS IN ADJUVANT THERAPY TREATED BY DENOSUMAB
}

SILVIA LEONE ${ }^{1}$, Alessandro de Sire ${ }^{2}$, Martina Ferrillo ${ }^{3}$, Filippo RENO' ${ }^{11}$, Marco Invernizzi ${ }^{1}$, Federica Pezzotti ${ }^{4}$, Mario Migliario $^{5}$, Sabrina Pasqua ${ }^{4}$

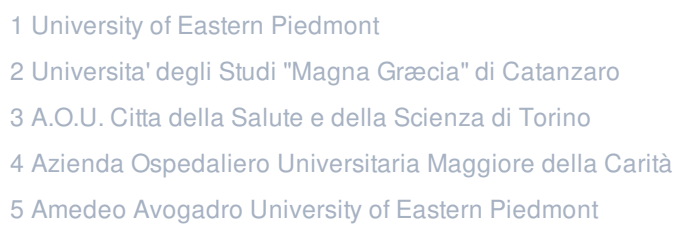

Funding: The author(s) received no specific funding for this work.

Potential competing interests: The author(s) declared that no potential competing interests exist.

\section{Abstract}

All patients ( $n=114)$ sents from Outpatient Clinic of Physical and Rehabilitative Medicine, "Maggiore della Carità" Hospital, Novara, Italy the underwent a dental examination, in anticipation of taking Denosumab. Patients undergoing a dental check-up were on adjuvant therapy with hormone therapy drugs due to previous breast and prostate cancer. Good oral health is a prerequisite for starting denosumab treatment. 
Background: Denosumab, a human monoclonal antibody directed against the receptor activator of nuclear factor- $\mathrm{k} \beta$ ligand (RANKL), is used for the treatment of patients with bone metastases of solid cancer or osteoporosis ${ }^{[1]}$. Recent reports have demonstrated that denosumab could induce osteonecrosis of the jaws $(\mathrm{ONJ})^{[2,3,4]}$. In Italy, this product is marketed in two preparations: Proliaâ (Amgen Inc., Thousand Oaks, CA), 60 mg subcutaneous injection every six months for the treatment of osteoporosis and to decrease bone resorption in men in androgen-deprivation therapy for prostate cancer; Xgevaâ (Amgen Inc., Thousand Oaks, CA), $120 \mathrm{mg}$ subcutaneous injection every four weeks for the prevention of skeletal complications in patients with bone metastases from breast cancer, prostate and other solid cancers, and for the treatment of unresectable giant cell tumors ${ }^{[5,6,7]}$. The effects of denosumab on turnover and bone mineral density are reversible in case of treatment interruption, taking into account that it does not deposit in bone, unlike the amino bisphosphonates ${ }^{[8]}$. Materials and methods: Cancer patients (men affected by prostate cancer and women affected by breast cancer during adjuvant therapy with Denosumab) referred to the Outpatient Clinic of Physical and Rehabilitative Medicine, "Maggiore della Carità" Hospital, Novara, Italy, were recruited at the Dental Clinic from January 2017 to July 2018 . Our investigation assessed patients' oral cavity health by collecting data related to: dental formula, decayed/missed/filled teeth (DMFT), dental prosthesis, plaque index (PI), gingival index (GI), and oral mucosal lesions presence.

Results: Patients included in the study were 114 subjects, mean aged $66.67 \pm 10.58$ years, $92 \%(n=105)$ women (mean aged $66.0 \pm 10.58$ years) in adjuvant therapy for breast cancer and $8 \%(n=9)$ men (mean aged $74.67 \pm 7.50$ years) for prostate cancer. Five patients $(4 \%)$ were edentulous; the remaining $96 \%$ $(\mathrm{n}=109)$ had $18.05 \pm 2.71$ teeth, a mean $\mathrm{Pl}=1.97 \pm 0.75$ and $\mathrm{Gl}=0.96 \pm 0.69 ; 29$ patients had $1.69 \pm 1.44$ decayed teeth and among these 11 needed extractions, 23 patients had 2.88+2.71 residual dental roots; 12 patients had prosthetic needs. Our patients underwent 97 professional oral hygiene sessions, 21 dental fillings, 33 tooth or root extractions, 3 removable prosthetic rehabilitations. All oral surgeries performed by observing a drug holiday period of denosumab intake and an antibacterial prophylaxis protocol, had no side effects.

Conclusions: We showed that $95 \%$ of the subjects investigated needed for dental care and oral surgery. Therefore, the necessity to take anti-resorption drugs might be considered as an opportunity to perform dental tests and treatments, observing the specific and recommended protocols in order to reduce the risk of ONJ.

\section{References:}

1. Lipton A, Jacobs I. Denosumab: benefits of RANK ligand inhibition in cancer patients. Curr Opin Support Palliat Care 2011;5(3):258-64.

2. Ramírez L, López-Pintor RM, Casañas E, et al. New non-bisphosphonate drugs that produce osteonecrosis of the jaws. Oral Health Prev Dent 2015;13(5):385-93.

3. Corrado A, Cantatore FP. The bisphosphonates: chemical characteristics, skeletal biological effects and extra-skeletal effects. Reum 2005;57(3):142-53.3.

4. Eleutherakis-Papaiakovou E, Bamias A. Antiresorptive treatment-associated ONJ. Eur J Cancer Care (Engl) 2017;26(6):1-11.

5. Bink KF. Analysis of denosumab on skeletal-related events in patients with advanced breast cancer. Clin J Oncol Nurs 2015;19(5):108-14.

6. Gartrell BA, Coleman R, Efstathiou E, et al. Metastatic prostate cancer and the bone: significance and therapeutic options. Eur Urol 2015;68(5):850-8.

7. Foroughi Moghadam MJ, Taheri S, Peiravian F. A systematic review of clinical practice guidelines for castration-resistant prostate cancer. Iran J Pharm Res 2018;17(Suppl):17-37.

8. Migliario M, Melle A, Foglio Bonda PL. Bisphosphonates and jaw osteonecrosis: clinical protocols for dental hygienists. Prevenzione \& Assistenza Dentale 2010;36:42-8. 